\title{
ON THE TERMS MASS AND WEIGHT
}

\author{
E. Robens ${ }^{1 *}$, P. Klobes ${ }^{2}$, D. Balköse ${ }^{3}$, S. Amarasiri and A. Jayaweera ${ }^{4}$ \\ ${ }^{1}$ Institut für Anorganische Chemie und Analytische Chemie der Johannes Gutenberg-Universität, Duesbergweg 10-14, D-55099 \\ Mainz, Germany \\ ${ }^{2}$ BAM Bundesanstalt für Materialforschung und -prüfung, Richard-Willstätter-Str. 11, D-12489 Berlin, Germany \\ ${ }^{3}$ Izmir Institute of Technology Faculty of Engineering Department of Chemical Engineering, Gulbah çeköyü Urla İzmir Turkey \\ ${ }^{4}$ School of Science and Technology, University of Teesside, Middlesbrough TS1 3BA, UK
}

\begin{abstract}
A short survey is given on mass units and recommendations on the proper use of the notations mass and weight. Whereas mass is an inertial physical quantity in classical mechanics, weight is a force due to the gravitational field and depending on the geographic situation.
\end{abstract}

Keywords: atomic mass, electron volt, mass, relative, specific, weight

\section{Introduction}

Units and systems of units are always subject to variations due to the requirements of their applications. The acceptance of a definition can last a long time. This may be observed for the important basic quantity in the present SI system of units [1], mass. The roots of the concept of mass can be traced back about 300 years [2]. In 1799 the kilogram prototype was made and the unit kilogram was defined [3]. Nevertheless, until now, consequences of that definition have not been completely accepted. A survey on mass units accepted by the Bureau International des Poids et Mesures (BIPM) [4] is given in Table 1.

\section{Definition of mass and weight}

Mass is a measure of the amount of material in an object, being directly related to the number and type of atoms present in the object. We have no sense for the quantity mass; however we do for the force of an accelerated body, e.g. for its weight within the gravitational field of the earth. Newton's second law [5] states that

$$
F=m a
$$

where $F$ is force acting on the body, $m$ is the mass of the body, $a$ is acceleration. The corresponding SI units are $N=\mathrm{kg} \mathrm{m} \mathrm{s}^{-2}, \mathrm{~kg}$, and $\mathrm{m} \mathrm{s}^{-2}$, respectively.

The weight, $W$, is defined as the force acting on a body with mass $m$ under gravitational acceleration $g_{n}$

$$
W=m g_{\mathrm{n}}
$$

Thus whilst mass is an unchanging quantity in classical mechanics, weight changes with gravita- tional acceleration. The weight of a body depends on the geographical situation on earth and will be different on moon and the other planets.

In 1948 as a result of the General Conference on Weights and Measures it was published:

Declaration on the unit of mass and on the definition weight; conventional value of $g_{n}(\mathrm{CR}, 70)$.

Taking into account the decision of the Comité International des Poids et Mesures of 15 October 1887 , according to which the kilogram has been defined as unit of mass:

Taking into account the decision contained in the sanction of the prototypes of the Metric System, unanimously accepted by the Conférence Générale des Poids et Mesures on 26 September 1889;

Considering the necessity to an end to the ambiguity which in current practice still exists on the meaning of the word weight, used sometimes for mass, sometimes for mechanical force;

The Conference declares:

- The kilogram is the unit mass; it is equal to the mass of the international prototype of the kilogram;

- The word 'weight' denotes a quantity of the same nature as a 'force': the weight of a body is the product of its mass and the acceleration due to gravity; in particular, the standard weight of a body is a product of its mass and the standard acceleration due to gravity;

- The value adopted in the International Service of Weights and Measures for the standard acceleration due to gravity is $980.665 \mathrm{~cm} \mathrm{~s}^{-2}$, a value already stated in the laws of some countries.

By means of a balance we measure the weight but the scaling indicates mass units. The gravitational 
acceleration depends on the geographical situation, but differences in the value of weight are almost insignificant in customary weighing. Thus in daily life the notation 'mass' will replace 'weight' only slowly or never. Furthermore 'weight' denotes the weight piece, which is a calibrated mass.

On the other hand, in science mass is an important physical quantity, which must be clearly distinguished from weight. Nevertheless, looking into the literature we find often the notation weight. For objects, which will never be weighed by a conventional balance in the gravitational field - atoms and molecules - this notation is still familiar. Indeed, the mass of such particles is determined either indirectly or by means of mass spectrometers, which measure the mass and not the weight.

In Einsteinian mechanics [6], the mass, $m$, of a body moving with velocity, $v$, is related to the rest mass, $m_{0}$, by the equation:

$$
m=\frac{m_{0}}{\sqrt{1-v^{2} / c^{2}}}
$$

where $c$ is the velocity of electromagnetic radiation. At ordinary velocities of terrestrial objects, $v$ is very small compared with $c$, and $m \approx m_{0}$. However, in an electron beam (for example in an electron microscope), where $v$ is much higher, the difference is significant, and the above relativistic correction is applicable in calculating its wavelength.

\section{Atomic mass}

An important unit for chemists is amount of molecules expressed in moles, based on Avogadro con- stant. Furthermore, it is based on the number of constituent particles (protons, electrons and neutrons) of an atom of carbon-12. The mole was confirmed in 1969 as a basic SI unit.

In the book 'The International System of Units (SI)' of the Bureau International des Poids et Mesures (BIPM) [1] we read:

'Atomic weights' and 'molecular weights' ... are in fact relative masses.

Physicists and chemists have ever since agreed to assign the value 12 , exactly, to the so-called atomic weight of the isotope carbon with mass number 12 (carbon-12, ${ }^{12} \mathrm{C}$ ), correctly called the relative atomic mass $A_{\mathrm{r}}\left({ }^{12} \mathrm{C}\right)$. The unified scale thus obtained gives the relative atomic and molecular masses, also known as the atomic and molecular weights, respectively.

In the IUPAC manual 'Quantities, Units and Symbols in Physical Chemistry' [7], the so-called 'Green book', it is concluded:

For historical reasons the terms 'molecular weight' and 'atomic weight' are still used. For molecules $M_{\mathrm{r}}$ is the relative molecular mass or 'molecular weight'. For atoms $M_{\mathrm{r}}$ is the relative atomic mass or 'atomic weight', and the symbol $A_{\mathrm{r}}$ may be used. $M_{\mathrm{r}}$ may also be called the relative molar mass, $M_{\mathrm{r}, \mathrm{B}}=M_{\mathrm{B}} / M_{\mathrm{u}}$, where $M_{\mathrm{u}}=$ mass (in $\mathrm{g}$ ) of 1 mole of carbon-12.

The term 'atomic weight' is being phased out slowly and being replaced by 'relative atomic mass', however 'standard atomic weights' have maintained its name [8].

The molecular mass of a substance, formerly also called molecular weight and abbreviated as $M W$, is the mass of one molecule of that substance, relative to the unified atomic mass unit $\mathrm{u}$ (equal to $1 / 12$ of the mass of one atom of carbon-12). This is distinct from

Table 1 Mass and mass related units

\begin{tabular}{llll}
\hline Name & Symbol & Definition & SI unit \\
\hline Number of entities (e.g. molecules, atoms, ions, formula units) & $N$ & & - \\
Amount of substance, amount, (chemical amount) & $n$ & $n_{\mathrm{B}}=N_{\mathrm{B}} / L$ & $\mathrm{~mol}$ \\
Avogadro constant & $L, N_{\mathrm{A}}$ & $6.02214179 \pm 0.00000030 \cdot 10^{23}$ & $\mathrm{~mol}^{-1}$ \\
Mass of atom, atomic mass & $m_{\mathrm{a}}, m$ & & $\mathrm{~kg}$ \\
Mass of entity (molecule, formula unit) & $m, m_{\mathrm{f}}$ & $\mathrm{kg}$ \\
Atomic mass constant & $m_{\mathrm{u}}$ & $m_{\mathrm{u}}=m_{\mathrm{a}}\left({ }^{12} \mathrm{C}\right) / 12=1 \mathrm{u}$ & $\mathrm{kg}$ \\
Dalton, unified atomic mass unit & $\mathrm{Da}, \mathrm{u}$ & $1 \mathrm{Da}=1 \mathrm{u}=1.66053886(28) \cdot 10^{-27}$ & $\mathrm{~kg}$ \\
Molar mass & $M$ & $M_{\mathrm{B}}=m / n_{\mathrm{B}}$ & $\mathrm{kg} \mathrm{mol}$ \\
Molar mass constant & $M_{\mathrm{u}}$ & $M_{\mathrm{u}}=m_{\mathrm{u}} N_{\mathrm{A}}$ & $\mathrm{kg} \mathrm{mol}^{-1}$ \\
Relative molecular mass (relative molar mass, molecular weight) & $M_{\mathrm{r}}$ & $M_{\mathrm{r}}=m_{\mathrm{f}} / m_{\mathrm{u}}$ & - \\
Relative atomic mass (atomic weight) & $A_{\mathrm{r}}$ & $A_{\mathrm{r}}=m_{\mathrm{a}} / m_{\mathrm{u}}$ & - \\
Molar volume & $V_{\mathrm{m}}$ & $V_{\mathrm{m}, \mathrm{B}}=V / n_{\mathrm{B}}$ & $\mathrm{m}^{3} \mathrm{~mol}^{-1}$ \\
Mass fraction & $w$ & $w_{\mathrm{B}}=m_{\mathrm{B}} / m_{\mathrm{i}}$ & - \\
Electronvolt & $\mathrm{eV}$ & $1 \mathrm{eV}=1.60217653(14) \cdot 10^{-19}$ & $\mathrm{~J}$ \\
Electronvolt/c & $\mathrm{eV} \mathrm{c}$ & $1.783 \cdot 10^{-36}$ & $\mathrm{~kg}^{-2}$ \\
\hline
\end{tabular}


the relative molecular mass of a molecule $M_{\mathrm{r}}$, which is the ratio of the mass of that molecule to $1 / 12$ of the mass of carbon-12 and is a dimensionless number. Molar masses are almost never measured directly. They may be calculated as the sum of the standard atomic weights included in the chemical formula. Salts consist of ions and therefore the value is notified as formula mass instead of a molecular mass.

The unified atomic mass unit $(\mathrm{u})$, or dalton $(\mathrm{Da})$, is a small unit of mass used to express atomic and molecular masses. It is defined to be one-twelfth of the mass of an unbound atom of ${ }^{12} \mathrm{C}$ at rest and in its ground state. The unit is convenient because one hydrogen atom has a mass of approximately $1 \mathrm{u}$, and more generally an atom or molecule that contains $N_{\mathrm{p}}$ protons and $N_{\mathrm{n}}$ neutrons will have a mass approximately equal to $\left(N_{\mathrm{p}}+N_{\mathrm{n}}\right) u$. Atomic masses are often written without any unit and then the unified atomic mass unit is implied. In biochemistry, particularly in reference to proteins, the term 'dalton' is often used. Because proteins are large molecules, they are typically referred to in kilodaltons, or ' $\mathrm{kDa}$ '. The unified atomic mass unit, or dalton, is not a SI unit of mass, although it is accepted for use with SI under either name. The symbol amu for atomic mass unit is obsolete.

The Avogadro constant $N_{\mathrm{A}} \approx 6.022 \cdot 10^{23} \mathrm{~mol}^{-1}$ is the number of 'entities' (usually, atoms or molecules) in 1 mole. (The pure value $6.022 \cdot 10^{23}$ is called Avogadro's number.) The Avogadro constant and the mole are defined so that one mole of a substance with atomic or molecular mass $1 \mathrm{u}$ have a mass of $1 \mathrm{~g}$. Most molecules consist of a mixture of molecular masses due to naturally occurring isotopes. For this reason these sorts of comparisons are more meaningful and practical using molar masses which are generally expressed in $\mathrm{g} \mathrm{mol}^{-1}$, not $\mathrm{u}$. The one-to-one relationship between daltons and $\mathrm{g} / \mathrm{mol}$ is true but in order to be used accurately calculations must be performed with isotopically pure substances or involve much more complicated statistical averaging of multiple isotopic compositions [9].

\section{The relation between electronvolt and the units of mass}

One electronvolt $\mathrm{eV}$ is a very small amount of energy widely used in solid state, nuclear and particle physics. It is based on a notional experiment as the amount of energy that could be gained by a single unbound electron when it would be accelerated through an electrostatic potential difference of one volt, in vacuo [10]. It is equal to one volt times the (unsigned) charge of a single electron $C_{\mathrm{el}}$ :

$$
\begin{gathered}
1 \mathrm{eV}=1 \mathrm{~J} \mathrm{C}^{-1} C_{\mathrm{el}}= \\
1.60217653(14) \cdot 10^{-19} \mathrm{~J} \approx 0.160 \mathrm{aJ}
\end{gathered}
$$

The unit electronvolt is accepted (but not encouraged) for use with SI. A single atom is such a small entity that to talk about its energy in joules would be inconvenient. But instead of taking the appropriate SI unit attojoule physicists (and chemists) have unfortunately chosen, arbitrarily, a non-conformist unit called an electronvolt $(\mathrm{eV})$. In mitigation, one can acknowledge the convenience of this unit when it comes to experimental measurement of ionization energies of elements, an important parameter for chemists. The experimentally measurable parameter is the potential difference (measured in volts) required to dislodge the electron from the atom, leading to the erroneous description of the energy as the 'ionisation potential'. Whereas ioniszation energies in $\mathrm{eV}$ are convenient numerical quantities, the energies for dislodging electrons from single atoms are very small. This can be overcome if ioniszation energies are expressed in $\mathrm{kJ} \mathrm{mole}^{-1}$, the recommended unit.

According to Einstein [6] the relation between mass (at rest) and energy is

$$
E=m c^{2}
$$

where $c$ is velocity of light $\mathrm{m} \mathrm{s}^{-1}$.

$$
\begin{gathered}
E / m=c^{2}=\left(299792458 \mathrm{~m} \mathrm{~s}^{-1}\right)^{2}= \\
89.875 \cdot 10^{15} \mathrm{~J} \mathrm{~kg}^{-1}=89.875 \mathrm{PJ} \mathrm{kg}^{-1}
\end{gathered}
$$

So $1 \mathrm{~g}$ of mass is equivalent to the energy of 89.9 terajoules $(\mathrm{TJ})$.

It is common in particle physics, where mass and energy are often interchanged, to use $\mathrm{eV} \mathrm{c}^{-2}$ as a unit of mass.

$$
1 \mathrm{eV} \mathrm{c}^{-2}=1.783 \cdot 10^{-36} \mathrm{~kg}
$$

\section{The terms relative and specific}

A quantity whose magnitude is additive for subsystems is called 'extensive', examples are mass and volume. A quantity whose magnitude is independent of the extent of the system is called 'intensive', examples are temperature and pressure. The adjective 'specific' before the name of an extensive quantity is often used to mean 'divided by mass' [7]. When the symbol for the extensive quantity is a capital letter, the symbol used for the specific quantity is often the corresponding lower case letter.

The term 'specific' should be used only if the quantity can be (and is) divided by the total mass or partial mass of the sample in question. If it is related to another item as in the case of atomic and molecular mass the respective notation is 'relative'. 
Likewise the term 'molar' before the name of an extensive quantity is used to denote 'divided by amount in moles'. Thus the heat capacity of a given quantity of a substance is the energy required to raise the temperature of that quantity of the substance by $1{ }^{\circ} \mathrm{C}$ (or $1 \mathrm{~K}$ ), measured in joules $\left(\mathrm{J} \mathrm{K}^{-1}\right)$. The specific heat capacity is this quantity divided by its mass, measured in $\mathrm{J} \mathrm{K}^{-1} \mathrm{~kg}^{-1}$. The molar heat capacity is the quantity divided by relative molar mass, measured in $\mathrm{J} \mathrm{K}^{-1} \mathrm{~mol}^{-1}$.

\section{Conclusions}

The mass as an inertial quantity of a body should be clearly distinguished from its weight, which is the product of mass and the acceleration due to gravity. Therefore, wt $\%$, atomic and molecular weight should be avoided and instead mass $\%$, relative atomic mass, relative molecular mass or relative molar mass and formula mass should be used. If electronvolt is used it would be appropriate to mention in addition the respective value in SI units because that would facilitate further assessments.

\section{References}

1 E. Göbel, I. M. Mills and A. J. Wallard, The International System of Units (SI), in Bureau International des Poids et Mesures, Sèvres, 2006

2 M. Gläser, The Concept of Mass, in Comprehensive Mass Metrology, in Kochsiek, M. and Gläser, M., (Eds.), Wiley-VCH, Berlin, 2000, pp. 16-47.

3 H. R. Jenemann, D. Hoffmann and H. Witthöfft, Eds, PTB, Braunschweig 1996, p. 183.

4 BIPM, Bureau International des Poids et Mesures, Pavillon de Breteuil, 92312 Sèvres cedex, France http://www.bipm.org/.

5 I. Newton, Philosophiae Naturalis Principia Mathematica, Ed., London 1687.

6 A. Einstein, Ann Phys., 17 (1905) 891.

7 E. R. Cohen, T. Cvitas, J. G. Frey, H. Bertil, K. Kuchitsu, R. Marquardt, I. Mills, F. Pavese, M. Quack, J. Stohner, H. L. Strauss, M. Takami and A. J. Thor, Quantities, Units and Symbols in Physical Chemistry, in Green book IUPAC, (Ed.) RSC Publishing, Cambridge 2007.

8 P. de Bievre and H. S. Peiser, Pure Appl. Chem., 64 (1992) 1535.

9 http://en.wikipedia.org/wiki/Atomic_Mass_Unit. 2008 Wikipedia.

10 http://en.wikipedia.org/wiki/Electronvolt 2008 Wikipedia.

DOI: $10.1007 / \mathrm{s} 10973-008-9353-\mathrm{z}$ 